\title{
PUBLISHING PLATFORM FOR AERIAL ORTHOPHOTO MAPS, THE COMPLETE STACK
}

\author{
J. Čepickýa ${ }^{\mathrm{a}}$, L. Čapek ${ }^{\mathrm{a}}$ \\ ${ }^{a}$ Cleerio s.r.o., 19000 Prague, The Czech Republic - (jachym.cepicky, ladislav.capek)@geosense.cz
}

Commission IV, WG IV/5

KEY WORDS: drone, eBee, aerial photos evaluation, surface situation map, engineering networks, road register, publishing, map application, stack, cloud

\begin{abstract}
:
When creating set of orthophoto maps from mosaic compositions, using airborne systems, such as popular drones, we need to publish results of the work to users. Several steps need to be performed in order get large scale raster data published. As first step, data have to be shared as service (OGC WMS as view service, OGC WCS as download service). But for some applications, OGC WMTS is handy as well, for faster view of the data. Finally the data have to become a part of web mapping application, so that they can be used and evaluated by non-technical users.

In this talk, we would like to present automated line of those steps, where user puts in orthophoto image and as a result, OGC Open Web Services are published as well as web mapping application with the data. The web mapping application can be used as standard presentation platform for such type of big raster data to generic user. The publishing platform - Geosense online map information system - can be also used for combination of data from various resources and for creating of unique map compositions and as input for better interpretations of photographed phenomenons. The whole process is successfully tested with eBee drone with raster data resolution $1.5-4 \mathrm{~cm} / \mathrm{px}$ on many areas and result is also used for creation of derived datasets, usually suited for property management - the records of roads, pavements, traffic signs, public lighting, sewage system, grave locations, and others.
\end{abstract}

\section{INTRODUCTION}

When creating orthophoto maps with hight resolution airborne systems, such as popular flying drones, we are creating large amount of data, which we need to serve to the end user.

In the web applications, as they are build nowadays, data have to be served using PNG (W3C, 2003) or JPEG (2016) formats. Orthophoto images are usually stored in the GeoTIFF (2000) format.

For this task, special server programs are used, which are serving the original data in new desired (web-enabled) raster format. Those programs (mapping servers, mapservers), are usually also capable to transform original raster orthophoto maps to target coordinate reference system.

User clients (or their dekstop and web applications) are interacting with the mapservers via standardized interface, preferably based on OGC OWS services (Open GeoSpatial Consortium Open Web Services), such as (for raster data) WMS (Web Map Service, OGC WMS 2006,) or OGC WCS service is build on top of them - tiled cache service (e.g. OGC WMTS - Web Map Tile Service, 2010).

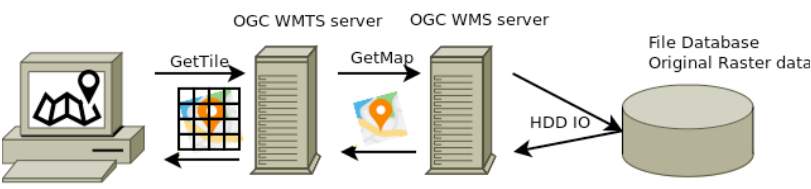

Figure 1: OGC OWS services - Client application requires predefined tiles, they are generated from another mapping service and cached on the WMTS server. WMS service is generated

Several steps need to be done, to get ortophoto map in mature spatial data infrastructure: shooting of the input photos, processing of the images to seem less true orthophoto mosaic, publishing the resulting image via web service, caching of the resulting images and last but not least describing the data and services with metadata in metadata catalogue.

\section{GEOSENSE ONLINE AIRBORNE IMAGERY MAP}

In Geosense company, whole production line is set and well tested for fast creation and publication of tiled raster view services.

\subsection{The scope}

Our partners and customers do require high precision orthophoto map serving as background for surveying and construction of technical maps of heigh detail. For this purpose, we need to build complete line from scratch - from production of input aerial images, over production of orthophoto imagery, to publishing the result on web applications and desktop programs, which are then used for processing of final data sets.

\subsection{Production of input data}

The whole process starts with taking pictures with semiautonomous drone device. Used eBee drone can cover up to 12 $\mathrm{km} 2$ in a single automated flight, while flights over smaller areas, flown at lower altitudes, can acquire images with a ground sampling distance (GSD) of down to $1.5 \mathrm{~cm}(0.6$ in) per pixel (Sensefly, 2016). The images are usually taken automatically - the operator just defines area which needs to be covered and optimal flight direction is chosen.

\subsection{Production of true orthophoto image}


The input raster images are then processed in PostflightTerra

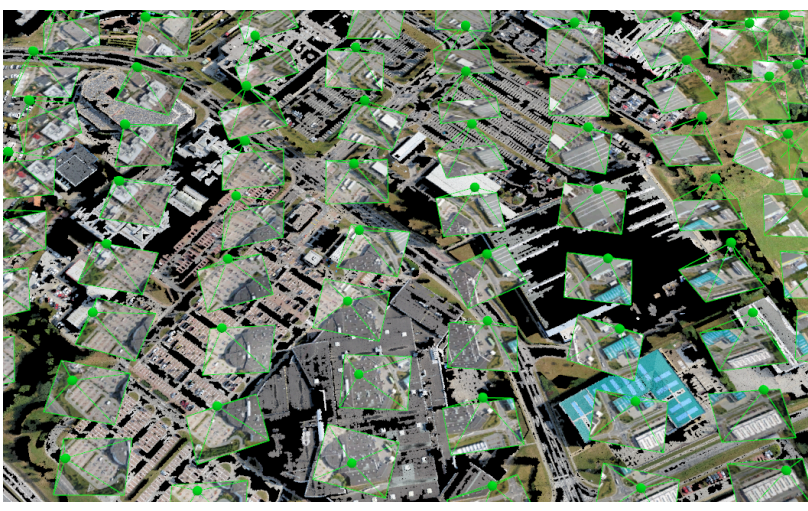

Figure 2: Poits of location and orientation of the camera while software (Postflight Terra, 2016). The output of the process is $3 \mathrm{D}$ point cloud data as well as $2 \mathrm{D}$ true orthophoto image, created as image mosaic from many input aerial photographs. Creating of the optimal seem less mosaic is usually done manually in the software. The operator has to choose carefully best fitting image for each mosaic tile with help of the software, so that no edges do occur on the final product.

The final orthophoto image is usually (depending on the area size) about $0.5 \mathrm{~GB}-2 \mathrm{~GB}$ big using the JPEG format (however, GeoTIFF images are stored too, as backup for later usage).

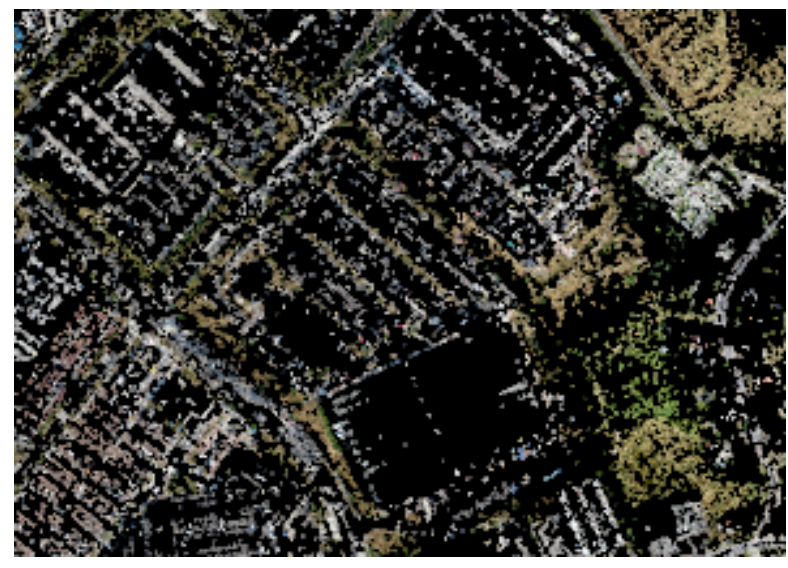

Figure 3: Point cloud of auto-corelated points with color

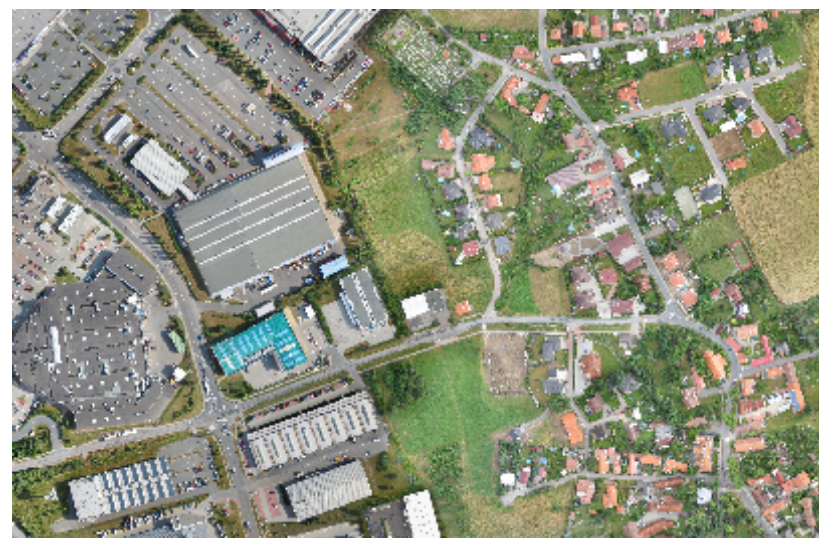

2.4 Setting up WMS server based on input orthophoto image data

For first step distribution of the aerial image, MapServer (2016) software is used. The server is set on two virtual machines, each equipped with 16 core Intel(R) Xeon(R) CPU E5-2695 v3@ $2.30 \mathrm{GHz}$ machine with 14GB of RAM. The main bottle necks now are IO operations, when accessing source raster files. After setting up the WMS server, WMTS cache server is prepared too.

For some applications this is the final product - we can serve the data using OGC WMS to various desktop programs as well as web applications. This is in particular useful, when arbitrary scale is required. Speed on the other hand is very slow, because whole raster image has to be processed, before being served.

\subsection{Setting up WMTS server for later creation of the tile cache}

As WMTS server, MapCache project of MapServer is used. After the server is set, automatic seeding script is deployed, which creates final raster data cache. This generated cache then is used as final service to the map application and other clients.

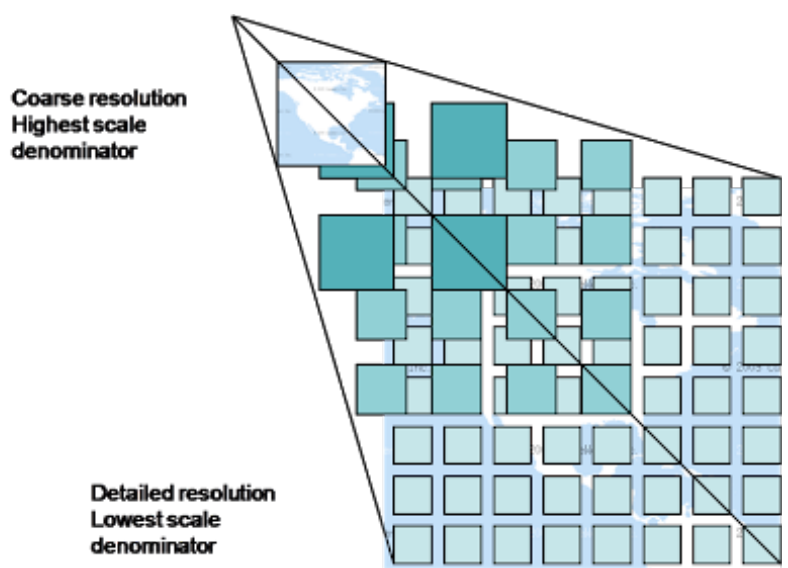

Since the input data are relatively big, after cache is created, we usually do remove the orthophoto image and disable WMS service - this enables us to use the hard drive capacity more efficient. Input orthophoto images are stored on separate backup hard drive. We can later re-create the tile cache from scratch.

The cache-seed process is running on server with 10 core Intel(R) Xeon(R) CPU E5-2695 v3 @ 2.30GHz CPU and 14 GB of RAM. Cache of the data itself is consuming around 900 GB of data.

Our system is able to produce tiles in various coordinate reference systems. For local partners, schema published by national State Administration of Land Surveying and Cadastre in their view services (CUZK, 2016) is used.

\subsection{Publishing platform}

The hardware used for final WMTS server uses SSD hard drive, but the resulting tile images are not stored on the naked file system, but they are served out of local file-based database. This will ensure lower load for the file systems and makes the backup easier. 


\subsection{Processing of the data}

After the image is published, new data can be derived communications, grave places, lightings, or e.g. database of private swimming pools.

This work is usually done manually, by educated operator. Some analysis can be done on semi-automatic manner, using e.g. GRASS GIS software (GRASS 2015).

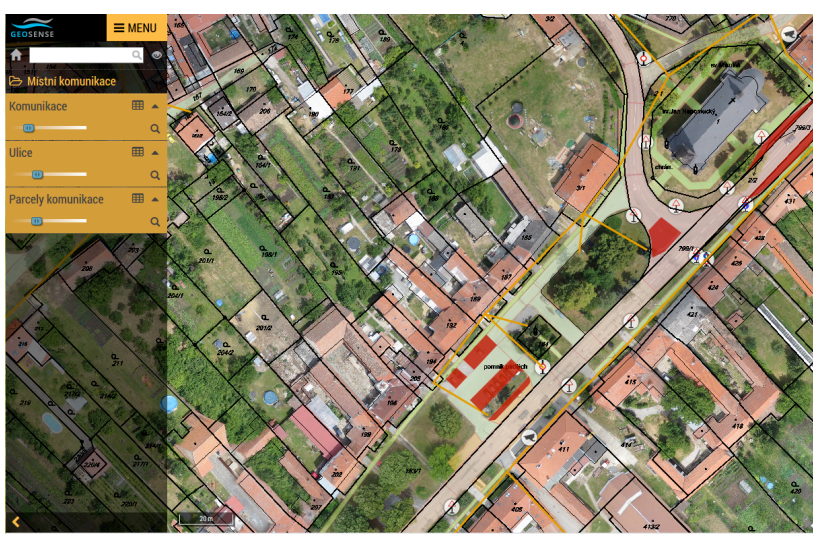

Figure 6: Geoportal application with technical map, communications and other data derived from detailed

\section{FURTHER STEPS}

We are now in the middle of process, which will lead to setting up complete spatial data infrastructure (SDI) - data are served with help of open web services. Those services will be described by metadata, which are going to be stored in the catalogue system. This will enable us keep track about services we are providing and help our partners to find out, data we are offering for their usage.

The derived data sets (technical maps, database of grave cuts and similar) can be den created based on published web services.

It is also our intention to offer the services to public community, especially editors of the OpenStreetMap (OSM 2016) project, who can re-use our data for further improvement of the OpenStreetMap project. Therefore we need to adjust our licence policy with ODbL (ODbL, 2016)

\section{REFERENCES}

W3C 2003. Portable Network Graphics (PNG) Specification (Second Edition), Information technology - Computer graphics and image processing - Portable Network Graphics (PNG): Functional specification. ISO/IEC 15948:2003 (E) https://www.w3.org/TR/PNG/ (06 May. 2016).

JPEG 2016, https://jpeg.org/, (6 May 2016).

GeoTIFF 2000, GeoTIFF Format Specification, GeoTIFF Revision 1.0, Dr. Niles Ritter (editor), http://www.remotesensing.org/geotiff/spec/geotiffhome.html, (6. May 2016)

OGC WMS 2006, OGC ${ }^{\circledR}$ OpenGIS ${ }^{\circledR}$ Web Map Server Implementation Specification 06-042, version 1.3.0, Jeff de la
Beaujardiere

(editor), http://www.opengeospatial.org/standards/wms (6. May 2016)

OGC WCS 2012, OGC ${ }^{\circledR}$ WCS 2.0 Interface Standard- Core: Corrigendum, OGC 09-110r4, vesion 2.0.1, Peter Baumann (editor), http://www.opengeospatial.org/standards/wcs (6. May 2016)

OGC WMTS 2010, OpenGIS ${ }^{\circledR}$ Web Map Tile Service Implementation Standard, OGC 07-057r7, version 1.0.0, : Joan Masó, Keith Pomakis and Núria Julià (editors), http://www.opengeospatial.org/standards/wmts (6. May 2016)

Sensefly 2016, eBee professional mapping drone, https://www.sensefly.com/drones/ebee.html (6. May 2016)

Pix4D 2016, https://pix4d.com/ (6. May 2016)

MapServer 2016, MapServer Project Steering Committee https://mapserver.org (6. May 2016)

CUZK 2016, WMTS Ortophoto view service, http://geoportal.cuzk.cz/WMTS_ORTOFOTO/WMTService.as px? service $=$ wmts\&request $=$ getcapabilities 9. May 2016

OSM 2016, OpenStreetMap project, http://openstreetmap.org, 9. Mab 2016

ODbL 2016, Open Data Commons Open Database License (ODbL), http://opendatacommons.org/licenses/odbl/ 9. May 2016

GRASS Development Team, 2015. Geographic Resources Analysis Support System (GRASS) Software, Version 7.0. Open Source Geospatial Foundation. http://grass.osgeo.org, 10. May 2016 